\title{
Notwendige Technik und logische Markterfordernisse einer Energiewende - Zwischenergebnisse des C/sells-Projektes
}

\author{
A. Reuter
}

Online publiziert am 18. November 2019

(c) Springer-Verlag GmbH Austria, ein Teil von Springer Nature 2019

C/sells ist ein Demonstrationsprojekt im Rahmen des SINTEGProgrammes. Das Förderprogramm „Schaufenster intelligente Energie - Digitale Agenda für die Energiewende" (SINTEG) des deutschen Bundesministeriums für Wirtschaft und Energie (BMWi) will skalierbare Musterlösungen für eine sichere, wirtschaftliche, umweltverträgliche und akzeptierte Energieversorgung bei hohen Anteilen fluktuierender Stromerzeugung aus Wind- und Sonnenenergie entwickeln und demonstrieren. Im Mittelpunkt stehen technische, wirtschaftliche und juristische Aspekte sowie die Integration der Bürger.

Wir haben die Grundkonzeption des zellulär verbundenen Energiesystems entwickelt und in den ersten C/sells-Zellen implementiert, die C/sells-Leitidee im Konsens der 56 Partner vereinbart und gelebt sowie die Grundbausteine unserer drei Basisinstrumente Infrastruktur-Informationssystem (IIS), Abstimmungskaskade und regionalisierter Handel erstellt und erprobt.

Die Stärkung der lokalen und regionalen Verantwortung, wohlgemerkt nicht der Autarkie, verleiht dem zellulär verbundenen Gesamtsystem Stabilität, Resilienz und gesellschaftliche Akzeptanz. Der damit einhergehende Umbau der technischen Infrastruktur und der Wandel der Geschäftsmodelle kann nach unserer Überzeugung nur mit der Bündelung aller gesellschaftlichen und politischen Kräfte gelingen. Unsere wesentlichen energiewirtschaftlichen Erkenntnisse fassen wir wie folgt stichwortartig zusammen:

- Zellularität: Wir sind überzeugt, dass zelluläre, vielfältige und partizipative Energieinfrastrukturen einen geeigneten Ansatz darstellen, um die angestrebte, nahezu vollständige Marktdurchdringung erneuerbarer Energien zu beherrschen und Versorgungssicherheit gewährleisten zu können. In Kooperation mit der Politik ist das Umfeld derart zu gestalten, dass den Zellteilnehmern jeweils ein möglichst hoher Autonomiegrad ermöglicht wird.

- Systemdenken: Die systemische Betrachtung zellulärer Systeme bietet im Vergleich zur Einzeltechnologie-Perspektive in vielfältiger Hinsicht Vorteile; bei der Systemauslegung, im Betrieb, im Marktverhalten und in Hinblick auf die Umweltwirksamkeit. In diese Richtung sollten die gesetzlichen Grundlagen ausgerichtet und die Zielerfüllung bewertet werden. Zudem ist eine Diskussion zu führen, die Gemeinwohl-, Verteilungsgerechtigkeits- und Entsolidarisierungsaspekte umfasst.

- Real-Labore: Angesichts des fundamentalen Wandels der Energiesysteme leistet $\mathrm{C} /$ sells einen bescheidenen Beitrag. Der vor uns liegende Weg wird Jahrzehnte dauern und ist in seiner Dimension mit keinem bisherigen Strukturwandel vergleichbar. Daher brauchen wir zeitlich unbefristete Experimentierräume, die intelligent vernetzt sind und neben Technik und Regulatorik auch den Markt, Safety \& Security, Resilienz, Governance und Partizipation erproben.

- Politisches Marketing: Das politische Marketing „pro Energiewende" bedarf einer massiven Verstärkung und Professionalisierung. So sind die mit den Energiewende-Gesetzen verbundenen intelligenten Messsysteme (iMSys) noch nicht durchgehend positiv besetzt, und die Vorteile einer gesicherten Kommunikationslinie sind für viele nicht ersichtlich. Ebenso ist politisches Marketing für Datenschutz, Datenvermeidung und Datensparsamkeit erforderlich. Jeder soll die Hoheit über seine Daten behalten, aber es muss auch vermittelt werden, dass für das Funktionieren des nachhaltigen Gesamtsystems gewisse Daten notwendig sind. Darüber hinaus fehlt eine durchgängige Argumentation für aktive Partizipation als Erfolgsfaktor der Energiewende. Der öffentliche Bereich sollte als Vorreiter fungieren und so mit gutem Beispiel vorangehen.

- Flexibilität: Wir verstehen Flexibilität als Stellschraube für das 100 \%-Szenario einer hoch volatilen Energiewelt. Die Flexibilitätsbereitstellung erproben wir nicht nur aus technischer Sicht, sondern auch mit marktlichen Ansätzen. Praxis: Letztendlich zählt die praktische Umsetzung in der Fläche. Unsere Praxiserfahrung in den C/sells-Zellen vor Ort zeigt, dass die Komplexität der Implementierungsarbeiten oftmals unterschätzt wird. Das Zusammenwirken vielfältiger Gewerke trat noch nie so massiv zutage wie bei der Umsetzung von digitalen Energiewendelösungen. Einen besonderen Schwerpunkt bilden der Liegenschaftsbereich und hier vor allem der Bestand.

Der Beitrag fasst die wesentlichen Ergebnisse der ersten 30 Projektmonate zusammen. Neben den wissenschaftlichen, technischen, systemischen und ökonomischen Erkenntnissen ist uns vor allem eines gelungen: Wir sind zu einer kreativen Gemeinschaft vielfältiger Akteure zusammengewachsen. Beweis hierfür ist der vorliegende Band, der die Beiträge unterschiedlichster Akteure zu einem Systemganzen vereint. Hieran beteiligen sich die Übertragungs- und Verteilnetzbetreiber, die Stadtwerke, die Hard- und Software-Lieferanten genauso wie Forschungseinrichtungen oder Start-ups. Diese im EcoSystem Vereinten werden auch nach Projektende weiter kooperieren und ihren Beitrag für die Implementierung der Energiewende leisten.

Hinweis des Verlags Der Verlag bleibt in Hinblick auf geografische Zuordnungen und Gebietsbezeichnungen in veröffentlichten Karten und Institutsadressen neutral.

Kurzfassung eines Vortrags bei der OVE-Energietechnik-Tagung, 57. OVE-/OGE-Fachtagun der Energietechnik-Branche, die am 16. und 17. Oktober 2019 in Innsbruck stattfand.

Reuter, Albrecht, Fichtner IT Consulting GmbH, Sarweystraße 3, 70191 Stuttgart, Deutschland (E-Mail: Albrecht.Reuter@fit.fichtner.de) 